TRANSACTIONS OF THE

AMERICAN MATHEMATICAL SOCIETY

Volume 184, October 1973

\title{
PSEUDO-DIFFERENTIAL ESTIMATES FOR LINEAR PARABOLIC OPERATORS
}

\author{
BY
}

\section{DAVID ELLIS}

ABSTRACT. In recent papers, S. Kaplan and D. Ellis have used singular integral operator theory, multilinear interpolation and forms of the classical "energy inequality" to obtain results for linear parabolic operators. For higher order linear parabolic operators the local estimates were globalized by a Gårding type partition of unity. In the present paper it is shown how the theory of pseudo-differential operators can be used to study linear parabolic operators without recourse to multilinear interpolation. We al so prove that the Gårding type partition of unity is square summable in the Sobolev type spaces $H^{S}$ and $K^{r, s}$.

1. Introduction. In [1] we studied differential operators of the form

$$
P\left(x, t, D_{x}, D_{t}\right)=\sum_{|a|+2 k j \leq 2 k m} a_{a, j}(x, t) D_{x}^{a} D_{t}^{j}
$$

with $a_{0, m}$ nonvanishing and the functions $\left\{a_{a, j}:|a|+a k j \leq 2 k m\right\}$ belonging to the class $C_{B}^{\infty}\left(R^{n+1}\right)$ of complex valued functions having bounded derivatives of all orders on $R^{n+1}$ (our notation is the same as that used in [1]). In addition, we assumed that $P$ was uniformly $2 k$-parabolic on $R^{n+1}$, i.e., there exists $\delta>0$ such that if

$$
P_{0}(x, t, \xi, z) \equiv \sum_{|a|+2 k j=2 k m} a_{a, j}(x, t) \xi^{a} z^{j}=0
$$

for $\langle x, t\rangle \in R^{n+1}$ and $\xi \in \Sigma=\left\{\xi \in R^{n}:|\xi|=1\right\}$, then $\operatorname{Im} z \geq \delta$. We assumed that $\delta$, a module of parabolicity for $P$, was fixed throughout.

By means of a change of variables we associated with $P$ an evolution operator $R=\partial / \partial t-H(t) \Lambda^{2 k}-J(t)$, where $H(t)$ on $J(t)$ were matrices of singular integral operators. We also saw that the eigenvalues of $b(t)$, the symbol of $H(t)$, were uniformly contained in a fixed compact subset of the open left-half complex plane. For such matrices as $b(t)$ we proved an important algebraic inequality (see Theorem 1 of [1]). Using this algebraic inequality and a Gărding type partition of unity on $R^{n+1}$ (denoted by $\left\langle\zeta_{i}\right\rangle_{1}^{\infty}$ ) we extended a form of the classical "energy inequality" for constant coefficient parabolic operators to an energy inequality for

Received by the editors December 1, 1972.

AMS (MOS) subject classifications (1970). Primary 35K30, 35S05; Secondary 46F10.

Key words and phrases. Pseudordifferential operator, evolution operator, Gårding type partition of unity, Sobolev space, energy inequality. 
our variable coefficient operator $R$.

As in [3] we employed the Hilbert spaces $\mathcal{H}^{r, s}, r, s$ real, and their quotient spaces $\mathcal{H}^{r, s}(\Omega), \Omega$ an open "slab" in $R^{n+1}$, since they are naturally tailored for parabolic operators. We also employed the maps $\mathbb{M}_{\rho, \sigma}, \rho, \sigma$ real, which are the natural isometric isomorphisms of $\mathcal{H}^{r, s}$ onto $\mathfrak{H}^{r-\rho, s-\sigma}$. In extending our "energy inequality" for $R$ to the $\mathcal{H}^{r, s}(\Omega)$ spaces we employed a proposition of S. Kaplan (Proposition 5 of [3]). This proposition essentially says that if $a \in C_{B}^{\infty}\left(R^{n+1}\right)$ then the commutator $\left[\pi_{r, s}, a \cdot\right]=\pi_{r, s} a \cdot-a \cdot \pi_{r, s}$ is smoothing in the space variable. We remark that Kaplan uses multilinear interpolation to prove this proposition. Finally, in Theorems 4 and 7 of [1] we showed that if $-\infty<a<b<+\infty$ and $s$ is a real number the mapping $\phi \leftrightarrow \mathcal{P}_{\phi}=\left\langle P \phi, \phi(a),(\partial / \partial t) \phi(a),(\partial / \partial t)^{2} \phi(a), \ldots\right.$, $\left.(\partial / \partial t)^{m-1} \phi(a)\right\rangle$ is

(i) one-to-one from $\mathcal{H}^{r, s}(\Omega)$ into $\mathcal{H}^{r-2 k m, s}(\Omega) \oplus H^{r+s-k} \oplus H^{r+s-3 k} \oplus \cdots \oplus$ $H^{r+s-(2 m-1) k}$, but

(ii) is onto for all $r>(2 m-1) k$ where $r$ is not an odd multiple of $k$ (which would seem to be a particularly unnatural restriction).

In this paper we will (i) develop a smoother calculus for the partition of unity $\left\langle\zeta_{i}\right\rangle_{1}^{\infty}$ on the $H^{s}$ and $\mathcal{H}^{(r, s}$ spaces (Propositions 1 and 3 ), (ii) express $R$ as a matrix of pseudo-differential operators and eliminate the restriction on $r$ in the Cauchy problem for $P$, (iii) present some remarks by $H$. Kumano-go concerning estimates on the commutator $\left[\pi_{r, s}, a \cdot\right]$ which obviate multilinear interpolation (Proposition 4).

2. The energy inequality.

Definitions. (i) For any real number $m$, we denote by $S_{\rho, \delta}^{m}\left(R^{n}\right), 0 \leq \delta<\rho \leq 1$, the set of all functions $p \in C^{\infty}\left(R^{n} \times R^{n}\right)$ which satisfy with constants $C_{a, \beta}$

$$
\left|\partial_{x}^{a} \partial_{\xi}^{\beta} p(x, \xi)\right| \leq C_{a, \beta} \lambda(\xi)^{m-\rho|\beta|+\delta|a|} \text { on } R^{n} \times R^{n}
$$

for all $\alpha, \beta$; here $\lambda(\xi)=\left\{1+|\xi|^{2}\right\}^{1 / 2} . S_{\rho_{0} \delta}^{m}\left(R^{n}\right)$ is a Fréchet space with the seminorms \|\|||$\|_{m, a, \beta}=\sup _{x, \xi}\left|\partial_{x}^{a} \partial_{\xi}^{\beta} p(x, \xi)\right| \lambda(\xi)^{\rho|\beta|-m-\delta|a|}$. Set $S^{m}=S_{1,0}^{m}\left(R^{n}\right)$.

(ii) For $p \in S_{\rho, \delta}^{m}\left(R^{n}\right)$ we define the operator $P=p(x, D)$ by

$$
P u(x)=(2 \pi)^{-n / 2} \int_{R^{n}} e^{i(x, \xi)} p(x, \xi) \tilde{u}(\xi) d \xi,
$$

where $\tilde{u}$ is the Fourier transform of $u \in S\left(R^{n}\right)$; we say $p$ is the symbol of the pseudo-differential operator $P$. If $u \in \mathcal{S}\left(R^{n}\right)$ then $P u \in \mathcal{S}\left(R^{n}\right)$, and for any real $s$ there exists $C=C_{s}$ such that

$$
\|P u\|_{s} \leq C\|u\|_{s+m} \text { for all } u \in S\left(R^{n}\right) \text {. }
$$

In reducing $P$ to first order in $t$ we express $P$ as $P=P_{0}+P_{1}$ where the principal part of $P$ is 


$$
P_{0}\left(x, t ; D_{x}, D_{t}\right)=D_{t}^{m}+\sum_{j=1}^{m} p_{j}\left(x, t ; D_{x}\right) D_{t}^{m-j},
$$

$p_{j}(x, t ; \xi)$ is homogeneous in $\xi$ of degree $2 k j$. Also $P_{1}\left(x, t ; D_{x}, D_{t}\right)=\sum_{j=1}^{m} q_{j}\left(x, t ; D_{x}\right) D_{t}^{m-j}$, where $q_{j}(x, t ; \xi)=\Sigma_{|a|<2 k j} a_{a, m-j}(x, t) \xi^{a}, j=1,2, \ldots, m$.

As in [1] we are led to study the evolution operator $R=(\partial / \partial t) I-H(t) \Lambda^{2 k}-$ $J(t)$. However, here $H(t), \Lambda$ and $J(t)$ are matrices of pseudo-differential operators whose symbols are given by

$$
b(x, t ; \xi)=\left(\begin{array}{cccc}
0 & 1 & & 0 \\
0 & 0 & 1 \cdot . \cdot 1 \\
-p_{m}(x, t ; \xi / \lambda(\xi))-\cdots-p_{1}(x, t ; \xi / \lambda(\xi))
\end{array}\right),
$$

$\lambda(\xi) l$, and

$$
j(x, t ; \xi)=\left(\begin{array}{cccc}
0 & \cdots & 0 & 0 \\
0 & \cdots & 0 & 0 \\
\frac{-q_{m}(x, t ; \xi)}{\lambda(\xi)^{2 k(m-1)}}-\cdots & \cdots-\frac{q_{2}(x, t ; \xi)}{\lambda(\xi)^{2 k}}-q_{1}(x, t ; \xi)
\end{array}\right),
$$

respectively, for $\langle x, t ; \xi\rangle \in R^{n+1} \times R^{n}$. We note that $b(x, t ; \xi)$ and $j(x, t ; \xi)$ belong to bounded subsets of $S^{0}$ and $S^{2 k-1}$, respectively, uniformly in $t \in R^{1}$. By definition of $\delta$ (a module of parabolicity for $P$ ) there exists a compact subset $\Delta$ of $\mathrm{C}$ having the property that $\Delta \subset\{z: \operatorname{Re} z \leq-\delta \sqrt{2}\}$, and that each $\langle x, t ; \xi\rangle \epsilon$ $R^{n+1} \times \Sigma$ the eigenvalues of $b(x, t ; \xi)$ are contained in $\Delta$.

Let $\pi(\Delta)$ denote the set of matrices of the form (2.1) whose eigenvalues are contained in $\Delta$. If we let $t=\delta / 2 \sqrt{2}$ in Theorem 1 of [1] and denote $n(b)_{\delta / 2 \sqrt{2}}$ by $n(b)$, then we can find constants $C_{i}=C_{i}(\Delta, \delta, m)>0, i=1,2$, such that for each $b \in \pi(\Delta)$ there exists a nonsingular matrix $n(b)$ satisfying

$$
\begin{gathered}
C_{2}|n(b) \zeta| \leq|\zeta| \leq C_{1}|n(b) \zeta|, \text { and } \\
\operatorname{Re}\left(n(b)^{-1} b n(b) \zeta, \zeta\right) \leq-(\delta / 4 \sqrt{2})|\zeta|^{2} \quad \text { for all } \zeta \in \mathbf{C}^{m}
\end{gathered}
$$

For $b \in \pi(\Delta)$ and $R(b)=(\partial / \partial t) I-b \Lambda^{2 k}-J(t)$ we have the "energy inequality": there exists $C=C(\delta)>0$ such that if $-\infty<a<b<+\infty$ then

$$
\begin{gathered}
\frac{C_{2}}{2}\|u(b)\|_{0}^{2}-\frac{C_{1}}{2}\|u(a)\|_{0}^{2}+\frac{C_{2} \delta}{8} \int_{a}^{b}\|u(t)\|_{k}^{2} d t+C_{2}(\lambda-C) \int_{a}^{b}\|u(t)\|_{0}^{2} d t \\
\leq \operatorname{Re} \int_{a}^{b}\left(n(b)^{-1}(R+\lambda l) u(t), n(b)^{-1} u(t)\right)_{0} d t
\end{gathered}
$$


for all $u \in\left\{C_{0}^{\infty}\left(R^{n+1}\right)\right\}^{m}$ and $\lambda>0$ with $C_{1}, C_{2}$ as above (here $\|u\|_{s}^{2}=(u, u)_{s}$ where $(u, v)_{s}=\int \lambda(\xi)^{2 s} \widetilde{u}(\xi) \bar{v}(\xi) d \xi$; see Lemma 2 of [1]).

At each point $\left(x_{0}, t_{0} ; \xi_{0}\right) \in R^{n+1} \times \Sigma$ we have an energy inequality for

$$
R\left(b\left(x_{0}, t_{0}, \xi_{0}\right)\right)=R+\left(H(t)-b\left(x_{0}, t_{0}, \xi_{0}\right)\right) \Lambda^{2 k}
$$

when applied to functions whose supports are concentrated near $\left\langle x_{0}, t_{0}\right\rangle$ (here $b$ is given by (2.1)). Thus, in order to obtain an energy inequality for $R$, it would seem natural to employ a partition of unity on $R^{n+1}$ and obtain an estimate on the norm of the "error" operator $H(t)-b\left(x_{0}, t_{0}, \xi_{0}\right)$. For part of this estimate we employed a variation of a classical theorem due to Kohn and Nirenberg (Theorem 5 of [4]). For our present method we employ Theorem 5.3 of [5] for symbols of class $S_{\beta \delta}^{0}\left(R^{n}\right)$. Since our technique requires that locally the operator norm of $H(t)$ $b\left(x_{0}, t_{0}, \xi_{0}\right)$ be "small" and since the Kumano-go theorem requires some control of $\lim \sup _{\xi}\left|b(x, t ; \xi)-b\left(x, t ; \xi_{0}\right)\right|$, we have taken $\xi$ "close" to $\xi_{0}$ and employed another partition of unity on $\Sigma$. Unlike the partition of unity on $\Sigma$ employed in [1], our present partition of unity should give rise to pseudo-differential operators.

Our symbol $b(x, t ; \xi)$ defined by $(2.1)$ is in $s^{0}$ for each $t$ but it does not satisfy inequality (3.15) of [1]. Thus we define

$$
b^{\sharp}(x, t ; \xi)=i\left(\begin{array}{ccc}
0 & 0 & 0 \\
0 & 0 & 1 \cdot . \cdot \cdot 1 \\
-p_{m}(x, t ; \xi /|\xi|)-p_{m-1}(x, t ; \xi /|\xi|)-\cdots-p_{1}(x, t ; \xi /|\xi|)
\end{array}\right)
$$

for $\langle x, t ; \xi\rangle \in R^{n+1} \times\left(R^{n} \sim\{0\}\right)$. Clearly $b^{\#}(x, t ; \xi) \in \pi(\Delta)$. As in [1], given a fixed small positive number $\eta$ we construct a sequence of cubes $\left\langle Q_{i}\right\rangle_{1}^{\infty}$ satisfying the properties:

(2.4) (i) they overlap in a manner that any fixed point in $R^{n+1}$ is contained in exactly $2^{n+1}$ distinct cubes except for points on $U_{i} \partial Q_{i}$, and

(ii) $\left\|b^{\#}(x, t ; \xi)-b^{\#}\left(x_{i}, t ; \xi\right)\right\|\left\langle\eta / 2, i=1,2, \ldots\right.$, for all $\langle x, t ; \xi\rangle \in R^{n+1}$ $\times \Sigma$ where $\left\langle x_{i}, t_{i}\right\rangle$ is the center of $Q_{i}$ and $x_{1}=0, t_{1}=0$.

Choose $\zeta \in C_{0}^{\infty}\left(Q_{1}\right)$ so that $0 \leq \zeta \leq 1$. For each $i$ let $\zeta_{i}$ be a translate of $\zeta$ satisfying $\zeta_{i} \in C_{0}^{\infty}\left(Q_{i}\right), \zeta_{1}=\zeta$, and $\Sigma_{i=1}^{\infty} \zeta_{i}^{2} \equiv 1$ on $R^{n+1}$. It is clear that for any nonnegative integer $m$ there is $C_{m}=C_{m}(\eta)$ satisfying

$$
\sum_{|a|+j \leq m} \sum_{i}\left|\partial_{x}^{a} \partial_{t}^{j} \zeta_{i}(x, t)\right|^{2} \leq C_{m}
$$

for all $\langle x, t\rangle \in R^{n+1}$.

For a fixed $\left\langle x_{i}, t_{i}\right\rangle$, the center of $Q_{i}$, and $\xi_{0} \in \Sigma$ we write 


$$
\begin{aligned}
& b(x, t ; \xi)-b^{\#}\left(x_{i}, t_{i} ; \xi_{0}\right) \\
& \quad=\left(b(x, t ; \xi)-b^{\#}\left(x, t ; \xi_{0}\right)\right)+\left(b^{\#}\left(x, t ; \xi_{0}\right)-b^{\#}\left(x_{i^{\prime}}, t_{i} ; \xi_{0}\right)\right) .
\end{aligned}
$$

When multiplied by $\zeta_{i}$ the second difference on the right side of (2.5) will be bounded in absolute value by $\eta / 2$. Since $(\xi / \lambda(\xi))^{\alpha}-(\xi /|\xi|)^{\alpha} \rightarrow 0$ as $|\xi| \rightarrow \infty$, estimating the first difference on the right side of (2.5) reduces to estimating the difference $b^{\sharp}(x, t ; \xi)-b^{\sharp}\left(x, t ; \xi_{0}\right)$ for large $|\xi|$ and $\xi /|\xi|$ near $\xi_{0}$.

Given the number $\eta>0$ above, there exists $\delta>0$ satisfying

$$
\left\|b^{\#}(x, t ; \xi)-b^{\#}\left(x, t ; \xi_{0}\right)\right\|<\eta / 2
$$

for all $\langle x, t\rangle \in R^{n+1}$ whenever $\left|\xi-\xi_{0}\right|<\delta$. Fixing $\xi_{0} \in \Sigma$ let $\Omega, \Omega^{\prime}, \Omega^{\prime \prime}$ be open neighborhoods of $\xi_{0}$ on $\Sigma$ satisfying $\Omega^{\prime \prime} \subset \subset \Omega^{\prime} \subset \subset \Omega=\left\{\xi \in \Sigma:\left|\xi-\xi_{0}\right|<\delta\right\}$. Let $B_{\rho}=\left\{\xi \in R^{n}:|\xi|<\rho\right\}$ and let $K^{\prime \prime}=\kappa^{\prime \prime} \cup B_{1 / 4}, K^{\prime}=\kappa^{\prime} \cup B_{1 / 2}, K=\kappa \cup B_{3 / 4}$ where $\kappa^{\prime \prime}, \kappa^{\prime}, \kappa$ are the open cones subtended by $\Omega^{\prime \prime}, \Omega^{\prime}, \Omega$, respectively, having their vertices at the origin. Let $O_{1}, O_{2}, \cdots, O_{r}$ be a set of rotations on $R^{n}$ for which $O_{1}^{-1}\left(\Omega^{\prime \prime}\right), \ldots, O_{r}^{-1}\left(\Omega^{\prime \prime}\right)$ is a covering of $\Sigma(r$ depends upon $\eta)$. Clearly we can find functions $\phi_{1}, \cdots, \phi_{r}$ and $\psi_{1}, \cdots, \psi_{r}$, satisfying

(i) $\phi_{j}, \psi_{j} \in C_{B}^{\infty}\left(R^{n}\right), 0 \leq \phi_{j} \leq 1,0 \leq \psi_{j} \leq 1$,

(ii) $\operatorname{supp} \phi_{j} \subset O_{j}^{-1}\left(K^{\prime}\right)$ and $\Sigma_{j=1}^{r} \phi_{j}^{2} \equiv 1_{j}$ on $R^{n}$,

(iii) $\operatorname{supp} \psi_{j} \subset O_{j}^{-1}(K)$ and $\psi_{j} \equiv 1$ on $O_{j}^{-1}\left(K^{\prime}\right)$.

Thus we conclude the following:

$$
\sup _{x, t} \varlimsup_{|\xi| \rightarrow \infty} \| \psi_{j}(\xi)\left(\left(b(x, t ; \xi)-b^{\#}(x, t ; \xi ;)\right) \| \leq \eta / 2\right.
$$

where $\xi_{j}=. O_{j}^{-1}\left(\xi_{0}\right), j=1, \ldots, r$. Also

$$
\sup _{x, t}\left\|\zeta_{i}(x, t)\left\{b^{\sharp}\left(x, t ; \xi_{j}\right)-b^{\#}\left(x_{i}, t_{i} ; \xi_{j}\right)\right\}\right\| \leq \eta / 2
$$

for $i=1,2, \ldots$, and $j=1, \ldots, r$. Since $\phi_{j}, \psi_{j} \in S^{0}$ and $\psi_{j} \equiv 1$ on $\operatorname{supp} \phi_{j}$ the pseudo-differential operators $\Phi_{j}=\phi_{j}(D)$ and $\Psi_{j}^{j}=\psi_{j}(D)$ satisfy

$$
\Phi_{j} \Psi_{j}=\Phi_{j}, \quad j=1, \cdots, r .
$$

Moreover $\Sigma_{i}\left(\zeta_{i}(t) u, \zeta_{i}(t) v\right)_{0}=(u, v)_{0}$ for $u, v \in H^{0}$, and $\Sigma_{j=1}^{r}\left(\Phi_{j} u, \Phi_{j} v\right)_{s}=(u, v)_{s}$ for $u, v \in H^{s}$. As in [1], the reader should bear in mind that the above construction and choice of $\left\langle Q_{i}\right\rangle,\left\langle\zeta_{i}\right\rangle,\left\langle\phi_{j}\right\rangle,\left\langle\psi_{j}\right\rangle$ and $r$ depends upon the number $\eta$ satisfying (2.4). Thus if $l$ is any positive integer we have, by Leibnitz' rule, that

$$
\left|\sum_{i}\left\|\zeta_{i}(t) u\right\|_{l}^{2}-C_{0}\|u\|_{l}^{2}\right| \leq C_{1}\|u\|_{l-1}^{2}
$$

for all $u \in C_{0}^{\infty}\left(R^{n}\right)$ where $C_{0}=C_{0}(l)>0$ and $C_{1}=C_{1}(l, \eta)$.

We now state a stronger version of Proposition 3 in [1]. Let $\left\langle\zeta_{i}\right\rangle_{1}^{\infty}$ be a se- 
quence of functions satisfying

(i) $\zeta_{i} \in C_{0}^{\infty}\left(R^{n}\right), i=1,2, \ldots$,

(ii) no point in $R^{n}$ belongs to the supports of more than $k_{n}$ of the $\zeta_{i}$ 's (here $k_{n}$ is a fixed positive integer),

(iii) $\sup _{x} \Sigma_{i}\left|\partial_{x}^{a} \zeta_{i}(x)\right|^{2}<\infty$ for each multi-index $a_{0}$

For such a sequence $\left\langle\zeta_{i}\right\rangle_{1}^{\infty}$ there is a positive integer $N=N(n)$ so that, for any $y \in R^{n}$, supp $\zeta_{i} \cap\left\{x \in R^{n}:|x-y| \leq 1\right\}$ is nonempty for at most $N$ of the $\zeta_{i}$ 's.

Proposition 1. Let $\zeta=\left\langle\zeta_{i}\right\rangle_{1}^{\infty}$ be a sequence of functions as described above and suppose $s$ is real. Then there exists $C_{n, s}>0$ satisfying

$$
\sum_{i}\left\|\zeta_{i} u\right\|_{s}^{2} \leq C_{n, s} N C_{\zeta, s}\|u\|_{s}^{2}, \quad u \in C_{0}^{\infty}\left(R^{n}\right)
$$

where $N$ is given above and

$$
C_{\zeta, s}=\sup _{i}\left\{\sum_{|a| \leq[|s|]+1} \sup _{x}\left|\partial_{x}^{a} \zeta_{i}(x)\right|^{2}\right\}
$$

(bere $|s|$ is the integral part of $s$ ).

Proof. See $\$ 4$.

Corollary. If $\Sigma_{i}\left|\zeta_{i}\right|^{2} \equiv 1$ on $R^{n}$, then $\Sigma_{i}\left\|\zeta_{i} u\right\|_{s}^{2}$ is equivalent to $\|u\|_{s}^{2}$, $u \in H^{s}$.

Proof.

$$
\begin{aligned}
|(u, v)| & =\left|\sum_{i}\left(\zeta_{i} u, \zeta_{i} v\right)\right| \\
& \leq \sum_{i}\left\|\zeta_{i} u\right\|_{s}\left\|\zeta_{i} v\right\|_{-s} \leq C \sum_{i}\left\|\zeta_{i} u\right\|_{s}\|v\|_{-s}, \quad u \in H^{s}, v \in H^{-s}
\end{aligned}
$$

Thus $\|u\|_{s}=\sup \left\{(u, v) \mid /\|v\|_{-s}: v \in H^{-s}, v \neq 0\right\} \leq C \Sigma_{i}\left\|\zeta_{i} u\right\|_{s}$ for $u \in H^{s}$.

As in [1] we let $b_{i j}=b^{\#}\left(x_{i}, t_{i} ; \xi_{j}\right), i=1,2, \ldots, j=1, \ldots, r$, where $b^{\#}(x, t ; \xi)$ is given by $(2.3)$. Define

$$
R^{i j}=\partial / \partial t-b_{i j} \Lambda^{2 k}-J(t)
$$

where the symbol of $J(t)$ is given by (2.2). Define $n_{i j}=n\left(b_{i j}\right)$.

Theorem 1 (see Theorem 2 of [1]). Let $R=\partial / \partial t-H(t) \Lambda^{2 k}-J(t)$ and let $-\infty<a<b<+\infty$. Then there exists constants $C^{\prime}(\delta)$ and $C^{\prime \prime}(\delta)>0$ satisfying

$$
\begin{gathered}
\frac{C_{2}}{2}\|u(b)\|_{0}^{2}-\frac{C_{1}}{2}\|u(a)\|_{0}^{2}+C^{\prime}(\delta) \int_{a}^{b}\|u(t)\|_{k}^{2} d t+C_{2}\left(\lambda-C^{\prime \prime}(\delta)\right) \int_{a}^{b}\|u(t)\|_{0}^{2} d t \\
\leq \sum_{i, j} \operatorname{Re} \int_{a}^{b}\left(n_{i j}^{-1} \zeta_{i}(t) \Phi_{j}(R+\lambda l) u(t), n_{i j}^{-1} \zeta_{i}(t) \Phi_{j} u(t)\right)_{0} d t
\end{gathered}
$$


for all $u \in\left\{C_{0}^{\infty}\left(R^{n+1}\right)\right\}^{m}$ and all $\lambda>C_{2}^{-1}$.

Remarks on proof. While the proof of Theorem 1 here employs the theory of pseudo-differential operators, it essentially parallels the proof of Theorem 2 in [1]. A perusal of the proof of Theorem 2 in [1] should convince the reader that the following revisions will suffice to prove Theorem 1 here:

1. An application of Theorem 2.3 of $[6]$ to $\left[\Phi_{j}, H(t)\right]$ shows that its operator norm as a mapping from $H^{-k-1}$ into $H^{-k}$ is bounded by a constant $C_{k}$ independent of $t$.

2. Applying (2.4), (2.6), (2.7), (2.8), and Theorem 5.3 of [5], we obtain, for arbitrary $\epsilon>0$,

$$
\frac{C_{2}}{2}\|u(b)\|_{0}^{2}-\frac{C_{1}}{2}\|u(a)\|_{0}^{2}+\frac{C_{2} \delta}{8} \sum_{i, j} \int_{a}^{b}\left\|\zeta_{i}(t) \Phi_{j} u(t)\right\|_{k}^{2} d t+(\lambda-C) \int_{a}^{b}\|u(t)\|_{0}^{2} d t
$$

$$
\begin{aligned}
\leq & \sum_{i, j} \operatorname{Re} \int_{a}^{b}\left(n_{i j}^{-1} \zeta_{i} \Phi_{j}(R+\lambda l) u, n_{i j}^{-1} \zeta_{i} \Phi_{j} u\right)_{0} d t \\
& +(\epsilon+\eta) C \int_{a}^{b}\|u(t)\|_{k}^{2} d t+C(\epsilon, \eta) \int_{a}^{b}\|u(t)\|_{0}^{2} d t .
\end{aligned}
$$

In [1] we employed an elementary inequality (Lemma 3 of [1]) to estimate $\Sigma_{i, j}\left\|\zeta_{i} \Phi_{j} u(t)\right\|_{k}^{2}$ from below. But by (2.9) we obtain

$$
\sum_{i, j}\left\|\zeta_{i}(t) \Phi_{j} u(t)\right\|_{k}^{2} \geq C_{0}\|u(t)\|_{k}^{2}-C_{1}\|u(t)\|_{0}^{2}
$$

where $C_{0}=C_{0}(k)$ and $C_{1}=C_{1}(k, \eta)>0$ are independent of $t$. Letting $\epsilon=\eta=$ $C_{3} / 3 C$ in (2.10) along with (2.11) completes the proof.

Remarks on the extension of the energy inequality to distributions. We refer the reader to [3] for the various properties of the $\mathcal{H}^{r, s}$ and $\mathcal{H}^{r, s}(\Omega)$ spaces.

Proposition 2 (see Proposition 5 of [1]). Let $p$ be a positive integer. Then $u \in \mathcal{H}^{r, s}$ if and only if $u$ bas a representation of the form

$$
u-u_{0}+\sum_{|a|=2 k p} D^{a} u_{a}+D_{t}^{p} u_{p}
$$

where $u_{0}, u_{a}, u_{p} \in \mathcal{H}^{r+2 k p, s}$ for $|a|=2 k p$, in sucb a way that $\|u\|_{r, s}$ is equivalent to

$$
\left\{\left\|u_{0}\right\|_{r+2 k p, s}^{2}+\sum_{|a|=2 k p}\left\|u_{\alpha}\right\|_{r+2 k p, s}^{2}+\left\|u_{p}\right\|_{r+2 k p, s}^{2}\right\}^{1 / 2}
$$

Proof. See [1]. 
As in [1] we write

$$
[\phi, \psi]=\int_{R^{n+1}} \phi(x, t) \bar{\psi}(x, t) d x d t, \quad \phi, \psi \in C_{0}^{\infty}\left(R^{n+1}\right),
$$

which when extended makes $\mathcal{H}^{r, s}$ and $\mathfrak{H}^{-r,-s}$ dual Hilbert spaces.

Now Proposition 6 of [1] stated the following: Suppose $\left\langle\zeta_{i}\right\rangle_{1}^{\infty}$ and $\left\langle\rho_{i}\right\rangle_{1}^{\infty}$ are sequences in $C_{0}^{\infty}\left(R^{n+1}\right)$ which satisfy the conditions that for each $\alpha$ and $j$

$$
\sup _{x, t} \sum_{i}\left|\partial_{x}^{a} \partial_{t}^{j} \zeta_{i}(x, t)\right|^{2}<\infty \text { and } \sup _{x, t} \sum_{i}\left|\partial_{x}^{a} \partial_{t}^{j} \rho_{i}(x, t)\right|^{2}<\infty \text {. }
$$

Then for every pair of real numbers $r$ and $s$ the form $\Sigma_{i}\left[\zeta_{i} \phi, \rho_{i} \psi\right], \phi, \psi \epsilon$ $C_{0}^{\infty}\left(R^{n+1}\right)$, extends in a unique way to a continuous sesquilinear form on $\mathcal{H}^{r, s} \times$ $\mathfrak{H}^{-r_{1}-s}$. The proof of this proposition relied on Calderón's multilinear interpolation theorem. However, we can develop a smoother calculus of such sequences $\left\langle\zeta_{i}\right\rangle_{1}^{\infty}$ as follows.

Let $\left\langle\zeta_{i}\right\rangle_{1}^{\infty}$ be a sequence of functions satisfying

(i) $\zeta_{i} \in C_{0}^{\infty}\left(R^{n+1}\right)$ for each $i=1,2, \ldots$,

(ii) no point in $R^{n+1}$ belongs to the supports of more than $l_{n+1}$ of the $\zeta_{i}$ 's (here $l_{n+1}$ is a fixed positive integer),

(iii) $\sup _{x, t} \Sigma_{i}\left|\partial_{x}^{\alpha} \partial_{t}^{j} \zeta_{i}(x, t)\right|^{2}<\infty$ for each multi-index $\alpha$ and each nonnegative integer $j$.

Proposition 3. Let $\zeta=\left\langle\zeta_{i}\right\rangle_{1}^{\infty}$ be a sequence of functions as described above and suppose $r$ and $s$ are real. Then there is a constant $C_{n, s}>0$ satisfying

$$
\sum_{i}\left\|\zeta_{i} u\right\|_{r, s}^{2} \leq C_{n, s} C_{\zeta}(p+2, s)\|u\|_{r, s}^{2}
$$

for all $u \in C_{0}^{\infty}\left(R^{n+1}\right)$, where $p$ is the unique nonnegative integer satisfying $|r|=$ $2 k p+2 k \theta, \theta \in[0,1)$, and

$$
C_{\zeta}(p, s)=\sup _{i}\left\{\sum_{0 \leq j \leq p ;|a| s[|s|]+2 k p} \sup _{x, t}\left|\partial_{x}^{a} \partial_{t}^{i} \zeta_{i}(x, t)\right|^{2}\right\} .
$$

Proof. See $\$ 4$.

Corollary. If $\Sigma_{i}\left|\zeta_{i}\right|^{2} \equiv 1$ on $R^{n+1}$, then $\Sigma_{i}\left\|\zeta_{i} u\right\|_{r, s}^{2}$ is equivalent to $\|u\|_{r, s^{*}}^{2}$

Proof. See the proof of the Corollary to Proposition 1.

In the proof of the generalized "energy inequality for distributions" (Theorem 3 of [1]) we applied Proposition 5 of [3] to estimate the boundedness of the commutator $\left[\pi_{0, \rho}, H\right], \rho=r+s-k$. In Proposition 5 of [3], S. Kaplan uses singular integral operator theory and multilinear interpolation to prove that (i) the operation 
of multiplication by $a \in C_{B}^{\infty}\left(R^{n+1}\right)$ is continuous on $\mathcal{H}^{r, s}$ and (ii) $\pi_{r, s} a \cdot-a \cdot \pi_{r, s}$ is a bounded mapping from $\mathcal{H}^{\rho, \sigma}$ into $\mathcal{H}^{\rho-r, \sigma-s+\theta_{\text {for }}}$ any real $\rho$ and $\sigma$ with $\theta<1$, H. Kumano-go has remarked that in the $L^{2}$ theory of pseudo-differential operators interpolation theory is generally unnecessary. In particular he employs the theory of pseudo-differential operators to show that $\pi_{r, s} a \cdot-a \cdot \pi_{r, s}$ is bounded from $\mathcal{H}^{\rho, \sigma}$ into $\mathcal{H}^{\rho-r, \sigma+1}$ (Proposition 4 in $\$ 4$ ).

The revisions in Theorem 4 of [1] (uniqueness in the Cauchy problem for $P$ ) are fairly obvious and are rather minor. The revisions in Lemma 5 of [1] are very similar to the revisions stated for Theorem 1 with our pseudo-differential operator $R$ replacing the singular integral operator $R$ in [1]. Thus,

Theorem 3. If $s$ is real, $r>k$, and $-\infty<a<b<+\infty$, the mapping $u\langle R u, u(a)\rangle$ is a topological isomorphism of $\left\{\mathcal{H}^{r, s}(\Omega)\right\}^{m}$ onto $\left\{\mathcal{H}^{r-2 k, s}(\Omega)\right\}^{m} \oplus\left\{H^{r-k+s}\right\}^{m}$, where $\Omega=\Omega_{a, b^{*}}$

3. Existence in the Cauchy problem for $P$. We assume that $-\infty<a<b<+\infty$. Let

$$
\Re u=\langle R u ; u(a)\rangle \text { and } \mathcal{P}_{\phi}=\left\langle P \phi ; \phi(a) ;(\partial / \partial t) \phi(a), \ldots,(\partial / \partial t)^{m-1} \phi(a)\right\rangle .
$$

Theorem 4 (compare with Theorem 9 of [1]). If $-\infty<a<b<+\infty$, $s$ is any real number and $r>(2 m-1) k$, then $\mathcal{P}$ mapping $\mathcal{H}^{r, s}(\Omega)$ into $\mathcal{H}^{r-2 k m, s}(\Omega) \oplus$ $H^{r+s-k} \oplus H^{r+s-3 k} \oplus \cdots \oplus H^{r+s-(2 m-1) k}$ bas a bounded inverse, where $\Omega=\Omega_{a, b}$.

Proof. Let $f \in \mathcal{H}^{r-2 k m, s}(\Omega)$ and $\phi_{1}, \phi_{2}, \ldots, \phi_{m}, \phi_{j} \in H^{r+s-(2 j-1) k}$, be given. We must find $\phi \in \mathcal{H}^{r, s}(\Omega)$ satisfying $\mathcal{P}_{\phi}=\left\langle f ; \phi_{1}, \ldots, \phi_{m}\right\rangle$. By Theorem 3 there exists a unique element $u \in\left\{\mathcal{H}^{r} 0, s(\Omega)\right\}^{m}$ satisfying

$$
R u=i\left(\begin{array}{c}
0 \\
\vdots \\
\dot{0} \\
l
\end{array}\right) \text { and } u_{j}(a)=(i)^{j-1} \Lambda^{2 k(m-j)} \phi_{j}, \quad j=1,2, \cdots, m \text {, }
$$

where $r_{0}=r+2 k(1-m)$. Let $\phi=\Lambda^{-2 k(m-1)} u_{1} \in \mathcal{H}^{r_{r} s}(\Omega)$. Since $D_{t} u_{j}=\Lambda^{2 k} u_{j+1}$, $j=1, \ldots, m-1$, one easily shows that

$$
D_{t}^{m} \phi=D_{t} u_{m} \quad \text { and } \quad D_{t}^{m-j} \phi=\Lambda^{2 k(i-j)} u_{m-j+1}, \quad j=1, \cdots, m \text {. }
$$

This immediately implies that $P \phi=f$. Also an easy calculation shows that $\phi_{j}=$ $(\partial / \partial t)^{j-1} \phi(a), j=1, \cdots, m$. Thus $\mathcal{P}$ is onto and by the open mapping theorem we are done. 
4. Technical details.

Proof of Proposition 1. Let $C_{s}^{\prime}$ denote a constant depending only on $\zeta=\left\langle\zeta_{i}\right\rangle$ and $s$ which is not necessarily the same in each occurrence. Let $P(s)$ be the statement in the proposition corresponding to the real number $s$.

(i) We shall first show that $P(s)$ is true for all $s \in(0,1)$. . By Lemma 2.6.1 of [2] it suffices to show that $\Sigma_{i}\left\|\zeta_{i} u\right\|_{s}^{2}$ is equivalent to

$$
\|u\|_{0}^{2}+C_{s} \iint \frac{|u(x)-u(y)|^{2}}{|x-y|^{n+2 s}} d x d y .
$$

By Lemma 2.6.1 of [2] we have that

$$
\begin{aligned}
\sum_{i}\left\|\zeta_{i} u\right\|_{s}^{2} \leq & \sum_{i}\left\|\zeta_{i} u\right\|_{0}^{2}+C_{s} \sum_{i} \iint_{\frac{\left|\zeta_{i}(x) u(x)-\zeta_{i}(y) u(y)\right|^{2}}{|x-y|^{n+2 s}}} d x d y \\
& \leq C_{0}\|u\|_{0}^{2}+2 C_{s} \sum_{i} \iint \frac{|u(x)-u(y)|^{2}\left|\zeta_{i}(x)\right|^{2}}{|x-y|^{n-2 s}} d x d y \\
& +2 C_{s} \sum_{i} \iint \frac{\left|\zeta_{i}(x)-\zeta_{i}(y)\right|^{2}}{|x-y|^{n+2 s}}|u(y)|^{2} d x d y \\
& =C_{0}\|u\|_{0}^{2}+C_{s}^{\prime}\|u\|_{s}^{2}+2 C_{s} \sum_{i} \iint \frac{\left|\zeta_{i}(x)-\zeta_{i}(y)\right|^{2}}{|x-y|^{n+2 s}}|u(y)|^{2} d x d y
\end{aligned}
$$

To estimate the integral in (4.1) we observe that

$$
\begin{aligned}
& \sum_{i} \underset{1<|x-y|}{\iint_{x-y}} \frac{\left|\zeta_{i}(x)-\zeta_{i}(y)\right|^{2}}{|x-y|^{n+2 s}}|u(y)|^{2} d x d y \\
& \quad \leq C_{s}^{\prime} \int_{1<|x-y|} \frac{d x}{|x-y|^{n+2 s}}\|u\|_{0}^{2} \leq C_{s}^{\prime}\|u\|_{s}^{2}
\end{aligned}
$$

By the definition of $N$ we have that

$$
\sum_{i}\left|\zeta_{i}(x)-\zeta_{i}(y)\right|^{2} \leq N \sup _{i}\left\{\sum_{j=1}^{n} \sup _{x}\left|\partial_{x_{j}} \zeta_{i}(x)\right|^{2}\right\}|x-y|
$$

for $|x-y|<1$. Thus

$$
\begin{aligned}
& \sum_{i} \iint \frac{\left|\zeta_{i}(x)-\zeta_{i}(y)\right|^{2}}{|x-y|^{n+2 s}}|u(y)|^{2} d x d y \\
& \quad \leq N C \int_{R^{n}}|u(y)|^{2} d y \int_{|x-y| \leq 1} \frac{d x}{|x-y|^{n-2+2 s}} \leq N C_{s} C_{\zeta, 0}\|u\|_{s^{\circ}}^{2}
\end{aligned}
$$


Also $P(0)$ and $P(1)$ trivially hold.

(ii) We now assert that if $P(s)$ is true for some $s \in[0, \infty)$ then $P(s+1)$ is true. Let $u \in H^{s+1}$, then

$$
\left\|\zeta_{i} u\right\|_{s+1}^{2} \leq\left\|\zeta_{i} u\right\|_{s}^{2}+2 \sum_{j=1}^{n}\left\|\left(\partial_{x_{j}} \zeta_{i}\right) u\right\|_{s}^{2}+2 \sum_{j=1}^{n}\left\|\zeta_{i}\left(\partial_{x_{j}} u\right)\right\|_{s}^{2}, \quad i=1,2, \ldots
$$

But since we assume that $P(s)$ holds and $[|s+1|]=[s]+1$ we have that

$$
\sum_{i}\left\|\left(\partial_{x_{j}} \zeta_{i}\right) u\right\|_{s}^{2} \leq C_{s} N C_{\zeta_{,}+1}\|u\|_{s}^{2}, \quad j=1, \ldots, n .
$$

Thus $\Sigma_{i}\left\|\zeta_{i} u\right\|_{s+1}^{2} \leq C_{s} N C_{\zeta, s+1}\|u\|_{s}^{2}$.

(iii) Finally we shall show that if $P(s)$ is true for some $s \in(-\infty, 0]$, then $P(s-1)$ is true. We know that $u \in H^{s-1}$ if and only if there are elements $u_{0}, u_{1}$, $\ddot{\cdots}, u_{n} \in H^{s}$ is such a way that $u=u_{0}+\sum_{j=1}^{n} D_{x_{j}} u_{j}$ with $\|u\|_{s-1}^{2}$ equivalent to $\Sigma_{j=0}^{n}\left\|u_{j}\right\|_{s^{\circ}}^{2}$. With $P(s)$ assumed to be true and $u$ having the above representation, we have that

$$
\begin{aligned}
\sum_{i}\left\|\zeta_{i} u_{0}\right\|_{s-1}^{2} \leq C_{s} N C_{\zeta_{0} s}\left\|u_{0}\right\|_{s}^{2}, \\
\sum_{i}\left\|\left(\partial_{x_{j}} \zeta_{i}\right) u_{j}\right\|_{s-1}^{2} \leq \sum_{i}\left\|\left(\partial_{x_{j}} \zeta_{i}\right) u\right\|_{s}^{2} \leq C_{s} N C_{\zeta_{0} s-1}\left\|u_{j}\right\|_{s}^{2}, \quad j=1, \cdots, n,
\end{aligned}
$$

since $[|s-1|]=[|s|+1]=[|s|]+1$. Also

$$
\sum_{i}\left\|D_{x_{j}}\left(\zeta_{i} u_{j}\right)\right\|_{s-1}^{2} \leq \sum_{i}\left\|\zeta_{i} u_{j}\right\|_{s}^{2} \leq C_{s} N C_{\zeta_{0} s}\left\|u_{j}\right\|_{s}^{2}, \quad j=1, \ldots, n .
$$

Thus,

$$
\sum_{i}\left\|\zeta_{i} u\right\|_{s-1}^{2} \leq C_{s} N C_{\zeta_{0} s-1} \sum_{j=0}^{n}\left\|u_{j}\right\|_{s}^{2} \leq C_{s} N C_{\zeta_{o} s-1}\|u\|_{s-1}^{2}
$$

(note that $C_{\zeta, s} \leq C_{\zeta, s-1}$ for $s \leq 0$ ). By combining (i), (ii), and (iii) the proof is complete.

Proof of Proposition 3. Let $C_{\zeta, r, s}$ denote a constant depending only on $\zeta=\left\langle\zeta_{i}\right\rangle_{1}^{\infty}, r$ and $s$ which is not necessarily the same in each occurrence. Let $P(r, s)$ be the statement of the proposition corresponding to the pair of real numbers $\langle r, s\rangle$.

(i) For each $t \in R^{1}$, no point in $R^{n}$ belongs to the supports of more than $l_{n+1}$ of the $\zeta_{i}(\cdot, t)$ 's. Thus by Proposition $2, P(0, s)$ holds for all $s \in R^{1}$.

Remark. $u \in \mathcal{H}^{r+2 k, s}$ if and only if $u_{t} \in \mathfrak{H}^{r, s}$ and $u \in \mathfrak{H}^{r, s+2 k}$; moreover, 


$$
\|u\|_{r+2 k, s}^{2}=\|u\|_{r, s}^{2}+\|u\|_{r, s+2 k}^{2} \leq 2\|u\|_{r+2 k, s}^{2}
$$

(ii) Let $r=2 k(p=1)$ and $s \in R^{1}$. Then by (4.2) and Proposition 1 we have

$$
\sum_{i}\left\|\zeta_{i} u\right\|_{2 k, s}^{2} \leq 2 \sum_{i}\left\|\left(\partial_{t} \zeta_{i}\right) u\right\|_{0, s}^{2}+2 \sum_{i}\left\|\zeta_{i} u_{t}\right\|_{0, s}^{2}+\sum_{i}\left\|\zeta_{i} u\right\|_{0, s+2 k}^{2}
$$

for all $u \in \mathcal{H}^{2 k, s}$.

(iii) Now suppose $P(r, s)$ is true for some $r \in(0, \infty)$ and all real $s$. We write $r=|r|=2 k p+2 k \theta$, where $p$ is a nonnegative integer and $\theta \in[0,1)$. As above we apply (4.2) and Proposition 1 to obtain

$$
\begin{aligned}
\sum_{i}\left\|\zeta_{i} u\right\|_{r+2 k, s}^{2} & \leq 2 \sum_{i}\left\|\left(\partial_{t} \zeta_{i}\right) u\right\|_{r, s}^{2}+2 \sum_{i}\left\|\zeta_{i} u_{t}\right\|_{r, s}^{2}+\sum_{i}\left\|\zeta_{i} u\right\|_{r, s+2 k}^{2} \\
& \leq C_{n, s} C_{\zeta}(p+2, s)\|u\|_{r+2 k, s}^{2}
\end{aligned}
$$

for all $u \in \mathcal{H}^{r+2 k, s}$. Thus $P(r+2 k, s)$ holds for all real $s$.

(iv) Now suppose $P(r, s)$ is true for some $r \in(-\infty, 0)$ and all real $s$. We write $r=2 k(-p)-2 k \theta$, where $p$ is a nonnegative integer and $\theta \in[0,1)$. By Proposition 2 we can write $u=u_{0}+\Sigma_{|a|=2 k} D^{a} u_{a}+D_{t} u_{1}$ with the elements $u_{0^{\prime}} u_{a^{\prime}} u_{1}$, $|a|=2 k$, chosen in $\mathcal{H}^{r, s}$ in such a way that $\|u\|_{r-2 k, s}^{2}$ is equivalent to $\left\|u_{0}\right\|_{r, s}^{2}+$ $\Sigma_{|a|=2 k}\left\|u_{a}\right\|_{r, s}^{2}+\left\|u_{1}\right\|_{r, s^{\circ}}^{2}$ Thus we can write

$$
\begin{aligned}
\sum_{i} \zeta_{i} u= & \sum_{i} \zeta_{i} u_{0}+\sum_{i}\left\{D^{\alpha}\left(\zeta_{i} u\right)-\sum_{0<\beta \leq \alpha}\left(\begin{array}{l}
\alpha \\
\beta
\end{array}\right) D^{\beta} \zeta_{i} \cdot D^{a-\beta} u_{\alpha}\right\} \\
& +\sum_{i}\left\{D_{t}\left(\zeta_{i} u_{1}\right)-\left(D_{t} \zeta_{i}\right) u_{1}\right\} .
\end{aligned}
$$

For $|a|=2 k$ and $0<\beta \leq \alpha$ we have that

$$
\left\|D^{\beta} \zeta_{i} \cdot D^{\alpha-\beta} u_{a}\right\|_{r-2 k, s} \leq\left\|D^{\beta} \zeta_{i} \cdot D^{\alpha-\beta} u_{\alpha}\right\|_{r, s-|\alpha-\beta|}
$$

By our assumption that $P(r, s)$ holds for all real $s$ we obtain

$$
\begin{aligned}
& \sum_{i}\left\|D^{\beta} \zeta_{i} \cdot D^{\alpha-\beta} u_{a}\right\|_{r, s-|\alpha-\beta|}^{2} \\
& \quad \leq C_{n, s}\left\{\begin{array}{l}
\sup _{i} \sum_{\substack{0 \leq j \leq p+2 \\
|\gamma| \leq[|s-| a-\beta \mid]+2 k(p+s)+|\beta|}} \sup _{x, t}\left|\partial_{x}^{\gamma} \partial_{t}^{j} \zeta_{i}(x, t)\right|^{2} \\
v^{2}
\end{array}\right)\left\|D^{\alpha-\beta} u_{a}\right\|_{r, s-|a-\beta|}^{2}
\end{aligned}
$$

Since $[|s-| \alpha-\beta||] \leq[|s|]+|\alpha-\beta|=[|s|]+2 k-|\beta|$, we have that 


$$
\begin{aligned}
\sum_{i}\left\|D^{\beta} \zeta_{i} \cdot D^{\alpha-\beta} u_{a}\right\|_{r, s-|\alpha-\beta|}^{2} & \leq C_{n, s} C_{\zeta}(p+2, s)\left\|D^{a-\beta} u_{a}\right\|_{r, s-|a-\beta|}^{2} \\
& \leq C_{n, s} C_{\zeta}(p+2, s)\left\|u_{a}\right\|_{r, s}^{2} .
\end{aligned}
$$

Applying Proposition 4 of $[3]$ and the assumption that $P(r, s)$ holds for all real $s$ to the remaining terms in (4.3) yields

$$
\begin{aligned}
\sum_{i}\left\|\zeta_{i} u\right\|_{r-2 k, s}^{2} & \leq C_{n, s} C_{\zeta}(p+2, s)\left\{\left\|u_{0}\right\|_{r, s}^{2}+\sum_{|a|=2 k}\left\|u_{a}\right\|_{r, s}^{2}+\left\|u_{1}\right\|_{r, s}^{2}\right\} \\
& \leq C_{n, s} C_{\zeta}(p+2, s)\|u\|_{r-2 k, s^{*}}^{2}
\end{aligned}
$$

Thus, if for some $r \in(-\infty, 0) P(r, s)$ holds for all $s \in R^{1}$, then $P(r-2 k, s)$ holds for all $s \in R^{1}$.

(v) If $r \epsilon(0,2 k)$ and $s$ is any real number than $u \in \mathcal{H}^{r, s}$ if and only if $u \in \mathcal{H}^{0, s+r}$ and

$$
I_{r, s}(u)=\int_{-\infty}^{\infty} \int_{-\infty}^{\infty} \frac{\|u(\theta)-u(\sigma)\|_{s}^{2}}{|\theta-\sigma|^{1+r / k}} d \theta d \sigma<\infty ;
$$

moreover, $\left\{\|u\|_{0, s+r}^{2}+I_{r, s}(u)\right\}^{1 / 2}$ is an equivalent norm for $\mathcal{H}^{r, s}$ (here $u(\theta)$ represents the function $x m \rightarrow u(x, \theta)$; see [3]).

Now let $r \in(0,2 k)$, in which case $p=0$, let $s$ be real and $u \in S\left(R^{n+1}\right)$. Then

$$
\begin{aligned}
\sum_{i}\left\|\zeta_{i} u\right\|_{r, s}^{2} & =\sum_{i}\left\|\pi_{0, s}\left(\zeta_{i} u\right)\right\|_{r, 0}^{2} \\
& \leq C_{r, s} \sum_{i}\left\|\pi_{0, s}\left(\zeta_{i} u\right)\right\|_{0, r}^{2}+C_{r, s} \sum_{i} I_{r, 0}\left(\pi_{0, s}\left(\zeta_{i} u\right)\right) .
\end{aligned}
$$

The first sum on the right side of (4.5) we estimate

$$
\begin{aligned}
& \sum_{i}\left\|\pi_{0, s}\left(\zeta_{i} u\right)\right\|_{0, r}^{2}=\sum_{i}\left\|\zeta_{i} u\right\|_{0, r+s}^{2} \\
& \quad \leq C_{r, s} C_{\zeta}(0, r+s)\|u\|_{0, r+s}^{2} \leq C_{r, s} C_{\zeta}(0, s)\|u\|_{r, s^{*}}^{2}
\end{aligned}
$$

The second sum on the right side of (4.5) we estimate 


$$
\begin{aligned}
& \sum_{i} I_{r, 0}\left(\pi_{0, s}\left(\zeta_{i} u\right)\right)=\sum_{i} \iint \frac{\left\|\left(\zeta_{i} u\right)(\theta)-\left(\zeta_{i} u\right)(\sigma)\right\|_{s}^{2}}{|\theta-\sigma|^{1+_{r} / k}} d \theta d \sigma \\
& \quad \leq 2 \sum_{i} \iint \frac{\left\|\zeta_{i}(\sigma)\{u(\theta)-u(\sigma)\}\right\|_{s}^{2}}{|\theta-\sigma|^{1+_{r} / k}} d \theta d \sigma+2 \sum_{i} \iint \frac{\left\|\left\{\zeta_{i}(\theta)-\zeta_{i}(\sigma)\right\}_{u}(\theta)\right\|_{s}^{2}}{|\theta-\sigma|^{1 t_{r} / k}} d \theta d \sigma \\
& \quad=2 I_{1}+2 I_{2}, \quad u \in S\left(R^{n+1}\right) .
\end{aligned}
$$

Clearly

$$
\begin{aligned}
I_{1} & \leq C_{n, s} C_{\zeta}(0, s) \iint \frac{\|u(\theta)-u(\sigma)\|_{s}^{2}}{|\theta-\sigma|^{1+r / k}} d \theta d \sigma \\
& \leq C_{n, s} C_{\zeta}(0, s)\|u\|_{r, s^{\circ}}^{2}
\end{aligned}
$$

By Proposition 1

$$
\sum_{i}\left\|\left\{\zeta_{i}(\theta)-\zeta_{i}(\sigma)\right\} u(\theta)\right\|_{s}^{2} \leq C_{n, s} C_{\zeta}(0, s)\|u(\theta)\|_{s}^{2} \text { for } u \in \mathcal{S}\left(R^{n+1}\right),
$$

which yields

$$
\begin{aligned}
& \sum_{i} \int_{1<|\theta-\sigma|} \int_{\theta} \frac{\left\|\left\{\zeta_{i}(\theta)-\zeta_{i}(\sigma)\right\} u(\theta)\right\|_{s}^{2}}{|\theta-\sigma|^{1+r / k}} d \theta d \sigma \\
& \quad \leq C_{n, s} C_{\zeta}(0, s) \int_{R}{ }^{1}\|u(\theta)\|_{s}^{2} d \theta \int_{1<|\sigma-\theta|} \frac{d \sigma}{|\sigma-\theta|^{1+r / k}} \\
& \quad \leq C_{n, r, s} C_{\zeta}(0, s)\|u\|_{0, s}^{2} \leq C_{n, r, s} C_{\zeta}(0, s)\|u\|_{r, s}^{2} \text { for } u \in S\left(R^{n+1}\right)
\end{aligned}
$$

As in Proposition 1 we can find a positive $N$ satisfying the property that, for any $y \in R^{n}, \operatorname{supp}\left(\zeta_{i}(\theta)-\zeta_{i}(\sigma)\right) \cap\left\{x \in R^{n}:|x-y| \leq 1\right\}$ is nonempty for at most $N$ of the functions $\zeta_{i}(\theta)-\zeta_{i}(\sigma)$ independent of $\theta$ and $\sigma$ where $|\theta-\sigma| \leq 1$. By Proposition 1 and the mean value theorem there exists $C_{n, s}>0$ satisfying the property that, for any $\theta, \sigma$ such that $|\theta-\sigma| \leq 1$,

$$
\begin{aligned}
& \sum_{i}\left\|\left\{\zeta_{i}(\theta)-\zeta_{i}(\sigma)\right\} u(\theta)\right\|_{s}^{2} \\
& \leq C_{n, s} N\left\{\sup _{i} \sum_{|a| \leq[|s|]+1} \sup _{x}\left|\partial_{x}^{a}\left\{\zeta_{i}(x, \theta)-\zeta_{i}(x, \sigma)\right\}\right|^{2}\right\}\|u(\theta)\|_{s}^{2} \\
& \quad \leq C_{n, s} N\left\{\sup _{i} \sum_{|a| \leq[|s|]+1} \sup _{x, t}\left|\partial_{x}^{a} \partial_{t} \zeta_{i}(x, t)\right|^{2}\right\}|\theta-\sigma|^{2}\|u(\theta)\|_{s^{\circ}}^{2}
\end{aligned}
$$

Thus we have that 


$$
\begin{aligned}
& \sum_{i} \iint \frac{\left\|\left\{\zeta_{i}(\theta)-\zeta_{i}(\sigma)\right\} u(\theta)\right\|_{s}^{2}}{|\theta-\sigma|^{1+r / k}} d \theta d \sigma \\
& \quad \leq C_{n, s} N C_{\zeta}(1, s) \int_{R}\|u(\theta)\|_{s}^{2} d \theta \int_{|\theta-\sigma| \leq 1} \frac{d \sigma}{|\theta-\sigma|^{r / k-1}} \\
& \quad \leq C_{n, r, s} N C_{\zeta}(1, s)\left\|_{u}\right\|_{r, s}^{2} \text { for } u \in S\left(R^{n+1}\right) .
\end{aligned}
$$

As a result, $I_{2} \leq C_{n, r, s} C_{\zeta}(1, s)\|u\|_{r, s}^{2}$ for $u \in \mathcal{S}\left(R^{n+1}\right)$ and we are done.

(vi) Now let $r \in(-2 k, 0)$, in which case $p=0$, and let $s$ be any real number. Again by Proposition 2 we can write $u=u_{0}+\Sigma_{|a|=2 k} D^{a} u_{a}+D_{t} u_{1}$ where the elements $u_{0}, u_{a}, u_{1},|\alpha|=2 k$, are chosen in $\mathcal{H}^{r+2 k, s}$ in such a way that $\|u\|_{r, s}^{2}$ is equivalent to $\left\|u_{0}\right\|_{r+2 k, s}^{2}+\Sigma_{|a|=2 k}\left\|u_{a}\right\|_{r+2 k, s}^{2}+\left\|u_{1}\right\|_{r+2 k, s^{-}}^{2}$. As before we express $\Sigma_{i} \zeta_{i} u$ in the form of $(4.3)$. Since $r+2 k=2 k(1-\theta) \epsilon(0, k)$ we can apply Proposition 1 to obtain

$$
\begin{gathered}
\sum_{i}\left\|\zeta_{i} u_{0}\right\|_{r, s}^{2} \leq \sum_{i}\left\|\zeta_{i} u_{0}\right\|_{r+2 k, s}^{2} \leq C_{n, s} C_{\zeta}(1, s)\left\|u_{0}\right\|_{r+2 k, s}^{2}, \\
\sum_{i}\left\|D^{a}\left(\zeta_{i} u_{a}\right)\right\|_{r, s}^{2} \leq \sum_{i}\left\|\zeta_{i} u_{a}\right\|_{r+2 k, s}^{2} \leq C_{n, s} C_{\zeta}(1, s)\left\|u_{a}\right\|_{r+2 k, s}^{2}
\end{gathered}
$$

for $|a|=2 k$, and

$$
\sum_{i}\left\|D_{t}\left(\zeta_{i} u\right)\right\|_{r, s}^{2} \leq \sum_{i}\left\|\zeta_{i} u_{1}\right\|_{r+2 k, s}^{2} \leq C_{n, s} C_{\zeta}(1, s)\left\|u_{1}\right\|_{r+2 k, s}^{2}
$$

Finally for $|\alpha|=2 k$ and $0<\beta<\alpha$, Proposition 1 yields

$$
\begin{aligned}
\sum_{i}\left\|D^{\beta} \zeta_{i} \cdot D^{a-\beta} u_{a}\right\|_{r, s}^{2} \leq \sum_{i}\left\|D^{\beta} \zeta_{i} \cdot D^{a-\beta} u_{a}\right\|_{r+2 k, s-|a-\beta|}^{2} \\
\quad \leq C_{n, s} C_{D} \beta_{\zeta}(2, s-|\alpha-\beta|)\left\|D^{\alpha-\beta} u_{a}\right\|_{r+2 k, s-|a-\beta|}^{2} \\
\quad \leq C_{n, s} C_{\zeta}(2, s)\left\|u_{a}\right\|_{r+2 k, s^{\circ}}^{2}
\end{aligned}
$$

Thus $\Sigma_{i}\left\|\zeta_{i} u\right\|_{r, s}^{2} \leq C_{\zeta}(2, s)\|u\|_{r, s}^{2}$ for $u \in \mathcal{H}^{r, s}$ and our proposition is proven. The following proposition is due to H. Kumano-go..

Proposition 4 (see Proposition 5 of [3]). Suppose a $\in C_{B}^{\infty}\left(R^{n+1}\right)$ is given. Then for every real $r$ and $s$

(i) $\pi_{r, s} a \cdot-a \cdot \pi_{r, s}$ is a bounded mapping from $\mathcal{H}^{\rho, \sigma}$ into $\mathcal{H}^{\rho-r, \sigma-s+1}$ for any real $\rho$ and $\sigma$, and

(ii) $a \cdot$ is a continuous mapping from $\mathcal{H}^{(r, s}$ into itself. 
Proof. Let $H^{-\infty}\left(R^{k}\right)=U_{s} H^{s}\left(R^{k}\right)$ and let $\mathcal{Q}^{-\infty}\left(R^{k}\right)$ denote the set of all linear operators from $H^{-\infty}\left(R^{k}\right)$ into $H^{-\infty}\left(R^{k}\right)$ which are infinitely smoothing. For $0 \leq \delta<\rho$ $\leq 1$ we let $\mathfrak{L}_{\rho, \delta}^{m}\left(R^{k}\right)$ denote the set of all linear operators $G: H^{-\infty}\left(R^{k}\right) \rightarrow H^{-\infty}\left(R^{k}\right)$ such that there exists a $p \in S_{\rho, \delta}^{m}\left(R^{k}\right)$ satisfying $G-p(x, D) \in \mathscr{L}^{-\infty}\left(R^{k}\right)$. Recall that

$$
Q(\xi, \tau)=\left\{\tau^{2}+\left(1+|\xi|^{2}\right)^{2 k}\right\}^{1 / 4 k} \text { and } \lambda(\xi)=\left\{1+|\xi|^{2}\right\}^{1 / 2}
$$

for $\xi \in R^{n}, r \in R^{1}$. Clearly $\lambda^{s} \in S_{1,0}^{s}\left(R^{n}\right)$ and $a \in S_{1,0}^{0}\left(R^{n+1}\right)$ for all $t \in R^{1}$ where $a(t)(x)=a(x, t)$.

With $[A, B]=A B-B A$ we have that

$$
\begin{aligned}
&\left.\| D \pi_{r, s}, a \cdot\right] u\left\|_{\rho-r, \sigma-s+1}=\right\| \pi_{\rho-r, 0} \Lambda^{\sigma-s+1}\left(\pi_{r, 0} \Lambda^{s} a \cdot-a \cdot \pi_{r, 0} \Lambda^{s}\right) u \|_{0,0} \\
& \leq\left\|\Lambda^{\sigma+1}\left[\pi_{\rho, 0}, a \cdot\right] u\right\|_{0,0}+\left\|\left[\Lambda^{\sigma+1}, a \cdot\right] \pi_{\rho, 0} u\right\|_{0,0} \\
&+\left\|\Lambda^{\sigma-s+1}\left[\pi_{\rho-r, 0}, a \cdot\right] \pi_{r, 0} \Lambda^{s} u\right\|_{0,0}+\|\left[\Lambda^{\sigma-s+1}, a \cdot \nabla \pi_{\rho, 0} \Lambda^{s} u \|_{0,0}\right. \\
&=I_{1}+I_{2}+I_{3}+I_{4} .
\end{aligned}
$$

By Corollary 2 of Theorem 4.1 of $[s]$ we have that $\left[\Lambda^{\sigma+1}, a(t) \cdot\right]$ and $\left[\Lambda^{\sigma-s+1}, a(t) \cdot\right]$ are uniformly bounded with respect to $t$ in $\mathscr{\varrho}_{1,0}^{\sigma}\left(R^{n}\right)$ and $\mathscr{L}_{1,0}^{\sigma-s}\left(R^{n}\right)$, respectively. Thus,

$$
I_{2} \leq C_{\sigma}\left\|\pi_{\rho, 0} u\right\|_{0, \sigma}=C_{\sigma}\|u\|_{\rho, \sigma}, \quad I_{4} \leq C_{\sigma}^{\prime}\left\|\pi_{\rho, 0} \Lambda_{u}^{s} u\right\|_{0, \sigma-s}=C_{\sigma}^{\prime}\|u\|_{\rho, \sigma} .
$$

To estimate $I_{1}$ and $I_{3}$ we employ Corollary 2 again:

$$
\mathbb{M}_{\rho, 0} a \cdot-a \cdot \mathbb{M}_{\rho, 0}=\sum_{0<|a| \leq N} \frac{1}{a !} a_{(a)} \mathbb{M}_{\rho, 0}^{(a)}+\mathcal{R}_{\rho, N},
$$

where $a_{(\alpha)}=\partial_{x, t}^{\alpha} a \cdot, M_{\rho, 0}^{(\alpha)}=\left(D_{\xi, t}^{\alpha} Q^{\rho}\right)\left(D_{x^{\prime}} D_{t}\right)$, and $R_{\rho, N} \in \mathscr{S}_{1 / 2 k}^{\rho_{0}-N / 2 k}\left(R^{n+1}\right)$ with $\rho_{0}=\max \{\rho, \rho / 2 k\}$. However the functions $\lambda^{\sigma+1} a_{(\alpha)}(t)$ are uniformly bounded in $S_{1,0}^{\sigma+1}\left(R^{n}\right)$ for $t \in R^{1}$; also $\left|D_{\xi, t}^{\alpha} Q^{\rho}(\xi, t)\right| \leq C_{\rho, a} Q(\xi, t)^{\rho-1}$ for $\alpha \neq 0$ and $\langle\xi, t\rangle \epsilon$ $R^{n+1}$. Thus

$$
\begin{aligned}
& \left\|\Lambda^{\sigma+1} a_{(a)} \pi_{\rho, 0}^{(a)} u\right\|_{0,0} \leq C_{a, \sigma}\left\|\pi_{\rho, 0}^{(a)} u\right\|_{0, \sigma+1} \\
& \quad \leq C_{a, \sigma}^{\prime}\|u\|_{\rho-1, \sigma+1} \leq C_{a, \sigma}^{\prime}\|u\|_{\rho, \sigma} \text { for } a \neq 0 .
\end{aligned}
$$

Now let us write

$$
\Lambda^{\sigma+1} R_{\rho, N}=\Lambda^{\sigma+1} M_{-(\sigma+1)^{\prime}, 0}\left[M_{(\sigma+1)^{\gamma}, 0} R_{\rho, N} M_{-\rho-\sigma^{\prime \prime}, 0}\right] M_{\rho, 0} M_{\sigma^{\prime \prime}, 0}
$$

where $(\sigma+1)^{\prime}=\max \{0, \sigma+1\}$ and $\sigma^{\prime \prime}=\min \{0, \sigma\}$. We observe that 


$$
\begin{aligned}
\lambda^{\sigma+1}(\xi) Q^{-(\sigma+1)^{\prime}}(\xi, t) & \leq C_{\sigma} \text { and } \\
Q^{\rho}(\xi, t) Q^{\sigma \prime \prime}(\xi, t) & \leq Q^{\rho}(\xi, t) \lambda^{\sigma}(\xi), \quad\langle\xi, t\rangle \in R^{n+1} .
\end{aligned}
$$

Also $\mathbb{M}_{(\sigma+1)^{\prime}, 0} R_{\rho, N} \mathbb{M}_{-\rho-\sigma_{0,0}^{\prime \prime}} \in \mathscr{L}_{1 / 2 k, 0}^{0}\left(R^{n+1}\right)$ for sufficiently large $N$. Hence we obtain

$$
\left\|\Lambda^{\sigma+1} R_{\rho_{0} N} u\right\|_{0,0} \leq C_{N}\left\|\pi_{\rho, 0} \pi_{\sigma^{\prime \prime}, 0} u\right\|_{0,0} \leq C_{N, \rho, \sigma}\|u\|_{\rho, \sigma}
$$

Combining (4.7) and (4.8) we obtain $I_{1} \leq C\|u\|_{\rho_{,} \sigma^{*}}$ If in (4.6) we replace $\rho$ by $\rho-r$ and $\sigma$ by $\sigma-s$ we obtain

$$
\left\|\Lambda^{\sigma-s+1} a_{(a)} \prod_{\rho-r, 0}^{(a)} \mathbb{M}_{r, 0} \Lambda^{s} u\right\|_{0,0} \leq C_{a, \sigma}\left\|\pi_{\rho-r, 0}^{(a)} \mathbb{M}_{r, 0} \Lambda^{s} u\right\|_{0,0}
$$

and

$$
\leq C_{a, \sigma}^{\prime}\|u\|_{\rho-1, \sigma+1} \leq C_{\alpha}^{\prime}\|u\|_{\rho, \sigma} \text { for } a \neq 0,
$$

$$
\begin{aligned}
\left\|\Lambda^{\sigma-s+1} R_{\rho-r, N} \pi_{r, 0} \Lambda^{s} u\right\|_{0,0} \leq C_{N}\left\|\pi_{\rho-r, 0} \pi_{(\sigma-s) \cdots, 0} \pi_{r, 0} \Lambda_{u}^{s} u\right\|_{0,0} \\
\leq C_{N, \rho, \sigma}\left\|\pi_{r, 0} \Lambda^{s} u\right\|_{\rho-r, \sigma-s}=C_{N, \rho, \sigma}\|u\|_{\rho, \sigma}
\end{aligned}
$$

for sufficiently large $N$. Combining (4.9) and (4.10) we obtain $I_{3} \leq C \|_{u, \sigma}$ and part (i) of the proposition is proven. That $a \cdot$ is bounded from $\mathcal{H}^{r,}, \bar{s}$ into itself is now obvious.

\section{REFERENCES}

1. D. Ellis, An energy inequality for higher order linear parabolic operators and its applications, Trans. Amer. Math. Soc. 165 (1972), 167-206.

2. L. Hörmander, Linear partial differential equations, Die Grundlehren der math. Wissenschaften, Band 116, Academic Press, New York; Springer-Verlag, Berlin, 1963. MR 28 \#4221.

3. S. Kaplan, An analogue of Gårding's inequality for parabolic operators and its applications, Michigan Math. J. 19 (1969), 269-305.

4. J. J. Kohn and L. Nirenberg, An algebra of pseudo-differential operators, Comm. Pure Appl. Math. 18 (1965), 269-305. MR 31 \#636.

5. H. Kumano-go, Algebras of pseudo-differential operators, J. Fac. Sci. Univ. Tokyo Sect. I 17 (1970), 31-50. MR 45 \#984.

6. ___ Remarks on pseudo-differential operators, J. Math. Soc. Japan 21 (1969), 413-439.

DEPARTMENT OF MATHEMATICS, HERBERT H. LEHMAN COLLEGE, CITY UNIVERSITY OF NEW YORK, BRONX, NEW YORK 10468 